\title{
Bothaella manhi, a new species of tribe Aedini (Diptera: Culicidae) from the Cuc Phuong National Park of Vietnam based on morphology and DNA sequence
}

\author{
SHELLEY COOK ${ }^{1}$, NGO GIANG LIEN $^{2}$, ERICA MCALISTER ${ }^{1} \&$ RALPH E. HARBACH ${ }^{1}$ \\ ${ }^{\prime}$ Department of Entomology, The Natural History Museum, Cromwell Road, London SW7 5BD, U.K. \\ E-mail: s.cook@nhm.ac.uk, e.mcalister@nhm.ac.uk,r.harbach@nhm.ac.uk \\ ${ }^{2}$ Department of Cell Biology, Hanoi University of Science, 334 Nguyen Trai Road, Hanoi, Vietnam. E-mail: ngogianglien@hus.edu.vn
}

\begin{abstract}
A new species of genus Bothaella (Diptera: Culicidae) collected along with two other species of the genus during surveys for flavivirus isolations in the Cuc Phuong National Park in northern Vietnam is formally described and named as Bothaella manhi, sp. n. The adults, pupa and fourth-instar larva are characterized, the male genitalia and the two immature stages are illustrated and DNA sequence data are included for regions coding for sections of the COI and COII genes (mtDNA). The species is compared and distinguished from the other species of the genus, and sequence data are used to hypothesise its phylogenetic relationship with Bo. helenae and Bo. kleini, the other two species collected during the survey.
\end{abstract}

Key words: Bothaella alongi, Bothaella helenae, Bothaella kleini, COI, COII, mosquito, new species, taxonomy

\section{Introduction}

Bothaella Reinert, 1973 is a little-known genus of mosquitoes (Diptera: Culicidae) that includes five species in Southeast Asia: Bo. alongi (Galliard \& Ngu, 1947), Bo. brownscutuma (Dong, Zhou \& Dong, 1999), Bo. eldridgei (Reinert, 1973), Bo. helenae (Reinert, 1973) and Bo. kleini (Reinert, 1973). Two of these species, Bo. helenae and Bo. kleini, and an unidentifiable species were found among adult mosquitoes collected in the Cuc Phuong National Park of Ninh Binh Province in northern Vietnam for flavivirus screening in August 2008. Additional specimens of Bo. helenae and the unidentifiable species were subsequently found in a collection of individually reared mosquitoes made in the park in July 2000. Dissection of the male genitalia confirmed that the unidentifiable species is distinct from Bo. brownscutuma, Bo. eldridgei, Bo. helenae and Bo. kleini, and a critical comparison of specimens with the detailed descriptions of the female and larva of $B o$. alongi (Reinert et al., 2006), the male of which is unknown, revealed it is distinct from that species as well. Based on these findings, the species is formally described and named as new in this paper. Sequence data for regions coding for sections of the COI and COII genes of mitochondrial DNA were obtained from specimens of the new species, Bo. helenae and Bo. kleini and used to hypothesise their phylogenetic relationships.

\section{Material and methods}

This study is based on wild-caught adults (vouchers of mosquitoes used for flavivirus screening) and adults reared from larvae and/or pupae collected in the Cuc Phuong National Park, Ninh Binh Province, northern Vietnam.

Morphology. Observations of adult mosquitoes were made under simulated natural light. Larval and pupal chaetotaxy were studied using differential interference contrast microscopy. Numbers in parentheses represent modes, when apparent, of the recorded ranges. The morphological terminology and abbreviations 
used in the descriptions and illustrations, respectively, are derived from the Anatomical Glossary of the online Mosquito Taxonomic Inventory (http://mosquito-taxonomic-inventory.info/anatomical-glossary-overview).

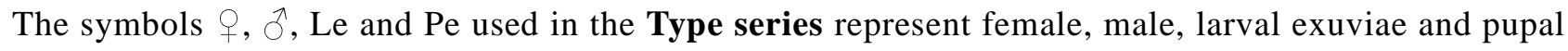
exuviae, respectively.

DNA sequences. Sequences of regions coding for sections of the COI and COII genes (mtDNA) were generated from adults and adults reared from larvae collected in the Cuc Phuong National Park. In addition to the three Bothaella species of particular interest from this specific locality, sequences were obtained from specimens representing a range of Culicidae collected in Thailand and Vietnam. One or more legs were taken from individual pinned mosquitoes that had been stored at $-80^{\circ} \mathrm{C}$ or dried over silica gel and homogenised as per Cook et al. (2006). A $60-\mu \mathrm{l}$ aliquot of the homogenate was added to $125 \mu \mathrm{l}$ of Chelex $100 \mathrm{Resin} 15 \% \mathrm{w} / \mathrm{v}$ (BioRad, Hercules, USA), incubated at $95^{\circ} \mathrm{C}$ for $20 \mathrm{~min}$ and centrifuged for $10 \mathrm{~min}$ at maximum speed. A 60 $\mu \mathrm{l}$ aliquot of supernatant was buffered using 10X PCR buffer (Roche Diagnostics, West Sussex, UK), and of this extraction $5 \mu 1$ was used in $25-\mu 1$ PCR reactions. All remaining homogenates and extractions were stored at $-80^{\circ} \mathrm{C}$ and $-20^{\circ} \mathrm{C}$, respectively.

The COI gene was amplified using primers LCO1490 (5'-GGTCAACAAATCATAAAGATATTGG-3') and HCO2198 (5'-TAAACTTCAGGGTGACCAAAAAATCA-3') (Folmer et al., 1994) with a primer concentration of $10 \mu \mathrm{M}$ and reaction conditions of $5 \mathrm{~min}$ at $95^{\circ} \mathrm{C}, 40 \mathrm{cycles}$ of $30 \mathrm{sec}$ at $95^{\circ} \mathrm{C}, 30 \mathrm{sec}$ at $48^{\circ} \mathrm{C}$, $45 \mathrm{sec}$ at $72^{\circ} \mathrm{C}$, followed by final extension time of $5 \mathrm{~min}$ at $72^{\circ} \mathrm{C}$. Identical reaction conditions were used to amplify the COII gene using the modified primers SCTL2-J-3037 (5'-ATGGCAGATTAGTGCAATGA-3') and TK-N-3785 (5'-GTTTAAGAGACCAGTACTTG-3') (Liu \& Beckenbach, 1992).

Only specimens from which clean forward and reverse sequences for both the COI and COII regions were obtained were included in phylogenetic analyses, with the exception of two specimens of Bo. kleini for which only COI sequence was obtained. Sequencher v. 4.8 (Gene Codes Corporation, Ann Arbor, USA) was used to combine reverse and forward sequences from each mosquito with primers trimmed. Final datasets were compiled using Se-Al (available at http://evolve.zoo.ox.ac.uk/software/Se-Al/main.html). Nucleotides were aligned using MUSCLE (Edgar, 2004). Maximum likelihood (ML) phylogenetic trees were estimated via Bayesian methods in MrBayes v. 3.1.2 (Huelsenbeck \& Ronquist, 2001) with a minimum of 50 million generations, a burnin of $10 \%$ and assessment of stationarity at effective sample size (ESS) $>400$ using Tracer v. 1.4.1 (part of the BEAST package, Drummond \& Rambaut, 2007). Sequences were submitted to GenBank (accession numbers are listed below).

\section{Taxonomy}

\section{Bothaella manhi Harbach \& Cook, new species}

Diagnosis. The adults of Bo. manhi have pale bands on the hindtarsi (distinction from Bo. kleini) and a patch of silvery scales on the postgena (distinction from Bo. alongi). The male of Bo. alongi is unknown, but the presence of a sigmoidal seta on the basal mesal lobe of the male genitalia distinguishes Bo. manhi from the other species of the genus. The pupa of Bo. manhi resembles the pupa of Bo. helenae and differs from the pupae of Bo. eldridgei and Bo. brownscutuma (pupae of Bo. alongi and Bo. kleini are unknown) in having a multiple-branched, stellate-like seta 1-Pa. The fourth-instar larva of Bo. manhi is distinguished from other members of the genus (the larva of Bo. kleini is unknown) by one or more of the following characters: seta 4$\mathrm{C}$ with 6 or fewer branches (distinction from Bo. alongi and Bo. eldridgei), setae 1-M,T and 1-VIII with 1-4 branches and seta 5-VIII single or double (distinctions from Bo. alongi and Bo. helenae) and siphon index greater than 4.0 (distinction from Bo. alongi, Bo. eldridgei and Bo. brownscutuma).

Sequences for the COI and COII regions of mtDNA distinguish Bo. manhi from Bo. helenae and Bo. kleini. Sequence data are unavailable for the other members of the genus, but the phylogenetic relationships inferred by the COI and COII sequences suggest these regions are likely to be diagnostic for each species.

Female. Overtly dark brown to black with areas of silvery-white scales. Head: Vertex with broad dark decumbent scales posteriorly and large triangular patch of broad silvery scales anteriorly that extend onto 
interocular space; occiput with transverse row of dark erect scales; postgena with small patch of silvery scales; ocular line narrow with broad dark scales, few dark ocular setae; 2 dark interocular setae. Compound eyes continuous above antennal pedicels. Clypeus bare. Antenna slightly longer than proboscis, pedicel with large mesal patch of broad silvery scales, flagellar whorls with relatively few setae, flagellomere 1 with dark scales mesally. Maxillary palpus length about $0.4 \mathrm{~mm}$, slightly less than 0.2 length of proboscis, dark-scaled. Proboscis narrow, length about $2.3 \mathrm{~mm}$, slightly longer than forefemur, dark-scaled. Thorax: Integument dark brown, scutum covered with narrow dark falcate scales except prescutellar area and supraalar area adjacent to posterior dorsocentral and prescutellar setae bare; dark setae on middle and lateral areas of anterior promontory, scutal fossa, prescutellar and antealar areas, anterior and posterior dorsocentral areas, supraalar area, lateral prescutellar line and 1 on parascutellar area; paratergite narrow, bare. Scutellum with broad silvery scales on midlobe, few broad dark scales on lateral lobes, several dark setae on all lobes. Mesopostnotum bare. Antepronotum with patch of broad silvery-white scales dorsally and line of dark setae anterior and lateral to scale patch. Postpronotum without scales, 2 or 3 dark setae posteriorly. Pleura with few dark setae: 2 upper proepisternal, few prealar, 2,3(3) mesokatepisternal, few upper and 1 lower mesepimeral; with prominent patches of broad silvery scales on upper proepisternal, postspiracular, upper mesokatepisternal, lower mesokatepisternal and upper mesepimeral areas. Wing: Length about $0.6 \mathrm{~mm}$; entirely dark-scaled; alula with row of narrow to slightly broad scales on margin; upper calypter with row of setae on upper margin; vein R2+3 slightly shorter than vein R2. Legs: Forecoxa with patches of broad silvery scales anteriorly at base and apex and dark scales adjacent to basal patch; mid- and hindcoxa with patch of silvery scales on anterior side of lateral midline; ventral surface of foretrochanter dark-scaled with silvery scales at apex, ventral surface of mid- and hindtrochanter with silvery scales; forefemur entirely dark-scaled except for inconspicuous silvery scales at apex of anterior surface, midfemur dark-scaled with small dorsoanterior patch of silvery scales at apex, proximal $0.5-0.6$ of hindfemur silvery-scaled, this area sometimes with few thinly scattered dark scales on dorsal midline, apex with dorsoanterior patch of silvery scales, dark scaling sharply contrasted between proximal and apical silvery areas; tibiae and foretarsus darkscaled, midtarsus with dorsal spots of white scales at bases of tarsomeres 1-3, hindtarsus with basal and apical bands of white scales on tarsomeres 1-4, tarsomere 5 white-scaled; fore- and midungues equal, each with 1 tooth, hindungues equal, both simple. Abdomen: Dark-scaled, laterotergite of tergum I with patch of silvery scales, terga II-VII with basolateral patches of silvery scales; sterna dark-scaled with basal bands of silvery scales confluent with basolateral patches of terga. Genitalia: Proximal 0.7 of tergum and sternum VIII retracted into segment VII, both wider than long; tergum VIII length $0.18 \mathrm{~mm}$, width $0.36 \mathrm{~mm}$, index 0.49 , posterior margin slightly concave, with setae and scales on caudal 0.4 , basolateral seta minute; sternum VIII length $0.28 \mathrm{~mm}$, width $0.38 \mathrm{~mm}$, index 0.75 , posterior margin produced on either side of midline, scales and setae on caudal 0.8 , setae denser and scales absent on posteromedian area, setae 1-5-S in more or less diagonal line. Tergum IX length $0.09 \mathrm{~mm}$, width $0.16 \mathrm{~mm}$, index 0.45 , posterior margin with shallow median emargination, 2-4 setae distally on either side of midline, 4-8 total setae; insula tongue-like, long, with 1-3 minute tuberculi distally. Postgenital lobe moderately long, apex with deep median emargination ( 0.36 of dorsal length), dorsal surface with 2 distal setae on either side of emargination, ventral surface with 5-7 setae on either side of midline, $14-18$ total setae, dorsal length $0.06-0.07 \mathrm{~mm}$, dorsal width $0.07 \mathrm{~mm}$, ventral length $0.12 \mathrm{~mm}$, ventral width $0.06 \mathrm{~mm}$, dorsal postgenital lobe index $0.86-1.08$, ventral postgenital lobe index 2.0, ventral postgenital lobe/cercus index 0.75 . Cercus moderately long, apex rounded, inner margin more or less straight, with setae on distal 0.4 of dorsal and lateral surfaces, scales absent, dorsal length $0.16 \mathrm{~mm}$, width $0.07 \mathrm{~mm}$, cercus index 2.29, cercus/dorsal postgenital lobe index 2.29-2.83. Three spherical spermathecal capsules, 1 slightly larger than the other two, few small spermathecal capsule pores near orifices.

Male. Similar to female except for obvious sexual differences; other differences include the following. Head: Proboscis slightly shorter, about $2.1 \mathrm{~mm}$, about 1.05 length of forefemur. Wing: Scaling reduced on veins posterior to R-R1. Genitalia (Fig. 1C): Tergum IX relatively broad, 2-5 setae on either side of midline (5-10 total setae), most lateral seta on either side inserted distinctly anterior to the others, which are usually in a more or less straight transverse line at posterior margin. Sternum IX large, with 3-7 (mode 5) setae inserted posteromedially. Gonocoxite relatively short and broad, mesal surface membranous, ventral, lateral and 
dorsomesal surfaces with numerous setae, dorsomesal setae generally thinner more flexible and in distinct patch, dorsolateral setae interspersed with scales, upper ventromesal area with dense patch of foliform setae (resembling scales but without longitudinal ridges). Gonostylus long, about 0.8 length of gonocoxite, apical 0.45 expanded with rounded apex, expanded portion with 3-5 dorsal setae and a single subapical ventrolateral seta; gonostylar claw tongue-like, borne apically. Basal mesal lobe expanded and elongate apically, with numerous tapered and lanceolate setae, caudal end of lobe slightly produced and bearing a lanceolate seta and a much longer flattened sigmoidal seta. Proctiger gradually tapered distally to blunt apex, cercus membranous, cercal setae absent; tergum X divided into 2 narrow sclerites borne laterally on either side at base of proctiger. Aedeagus comprised of 2 lateral, apically joined sclerites, ventral margins of sclerites thickened, ridge-like and weakly denticulate distally.

Pupa. Character and positions of setae as illustrated in Fig. 1A,B; numbers of branches in Table 1. Cephalothorax: Lightly tanned, scutum and metanotum darker; setae 3,6-CT very long and normally single. Trumpet: Angusticorn, more or less tubular, slightly bent anteriorly at tracheoid, moderately tanned, pinna without slit; length $0.52-0.73 \mathrm{~mm}$ (mean $0.61 \mathrm{~mm}$ ); pinna fairly long, 0.11-0.24 mm (mean $0.17 \mathrm{~mm}$ ); width at midlength $0.07-0.15 \mathrm{~mm}$ (mean $0.11 \mathrm{~mm}$ ); index 4.40-8.57 (mean 5.89). Abdomen: Length 2.70-4.21 mm (mean $3.35 \mathrm{~mm}$ ); lightly to moderately tanned, tergum I and anterior margins of terga and sterna II-VIII darker. Setae 3-I-III, 6-I and 5-IV,V very long, single (1 of 20 seta 6-I double), about twice length of following tergum; seta 2-II-VII normally inserted distinctly anteromesad of seta 1; seta 6-VII inserted more or less directly anterior to seta 9; seta 9-VII,VIII strongly developed, longer than segment VIII, with stiff aciculate branches; seta 10-II absent; seta 11-I usually single, occasionally double, often absent; seta 13-I usually absent or represented by an empty alveolus, minute and single if present. Genital lobe: Length about $0.2 \mathrm{~mm}$ in female; about $0.3 \mathrm{~mm}$ in male. Paddle: Longer than broad, ovoid, length $0.66-0.83 \mathrm{~mm}$ (mean 0.76 $\mathrm{mm}$ ), width $0.40-0.51 \mathrm{~mm}$ (mean $0.45 \mathrm{~mm}$ ), index 1.60-1.82 (mean 1.70); midrib long, distinct to seta 1-Pa; outer margin minutely spiculate on distal 0.75 , inner margin minutely spiculate on distal 0.25 . Seta $1-\mathrm{Pa}$ relatively long, more or less stellate, with 9-13(10) minutely aciculate branches; seta 2-Pa absent.

TABLE 1. Range (mode) of branches for pupal setae of Bothaella manhi obtained from 10 pupal exuviae (20 setae).

\begin{tabular}{|c|c|c|c|c|c|c|c|c|c|c|c|}
\hline \multirow{2}{*}{$\begin{array}{l}\text { Setae } \\
\text { no. }\end{array}$} & \multirow{2}{*}{$\begin{array}{l}\text { Cephalo- } \\
\text { thorax } \\
\text { CT }\end{array}$} & \multicolumn{9}{|c|}{ Abdominal segments } & \multirow{2}{*}{$\begin{array}{l}\text { Paddle } \\
\mathrm{Pa}\end{array}$} \\
\hline & & I & II & III & IV & $\mathrm{V}$ & VI & VII & VIII & IX & \\
\hline 0 & - & - & 1 & 1 & 1 & 1 & 1 & 1 & 1 & - & - \\
\hline 1 & $1,2(1)$ & $7-14(11)^{\mathrm{a}}$ & $1-4(2)$ & $1,2(1)$ & $1,2(1)$ & $1,2(1)$ & $1,2(1)$ & $1,2(1)$ & - & - & $9-14(10)$ \\
\hline 2 & $1,2(1)$ & $1-3(1)$ & $1,2(1)$ & $1,2(1)$ & $1-3(1)$ & $1,2(1)$ & $1-3(1)$ & 1 & - & - & - \\
\hline 3 & $1,2(1)$ & 1 & 1 & 1 & $2-5(4)$ & $1,2(1)$ & $1,2(1)$ & $1-3(2)$ & - & - & - \\
\hline 4 & $2-5(3)$ & $4-7(5,6)$ & $4-7(6)$ & $2-4(3)$ & $2-4(2)$ & $3-6(4)$ & $2,3(2)$ & $1,2(1)$ & $1,2(1)$ & - & - \\
\hline 5 & $2-5(3)$ & $1-3(2)$ & $1,2(1)$ & $1-3(2)$ & 1 & 1 & $1,2(1)$ & $1,2(1)$ & - & - & - \\
\hline 6 & 1 & $1,2(1)$ & $1,2(1)$ & 1 & $1,2(1)$ & 1 & $1-3(1)$ & $3-7(4)$ & - & - & - \\
\hline 7 & $1-4(2)$ & $1-4(2)$ & $2-4(3)$ & $2-7(3)$ & $2-5(3)$ & $4-6(5)$ & $1-3(2)$ & $1,2(1)$ & - & - & - \\
\hline 8 & $1-4(1)$ & - & - & $2-4(3)$ & $2-4(3)$ & $2-4(2)$ & $2-5(3)$ & $2-5(3)$ & - & - & - \\
\hline 9 & $1-3(1)$ & $1,2(1)$ & 1 & 1 & 1 & 1 & 1 & $4-9(8)$ & $8-13(9)$ & - & - \\
\hline 10 & $1-3(1)$ & - & $0-2^{\mathrm{d}}$ & $2,3(2)$ & $1-5(2)$ & $1,2(1)$ & $1,2(1)$ & $1-3(1)$ & - & - & - \\
\hline 11 & $1,2(1)$ & $0-2(1)^{\mathrm{b}}$ & $0-2^{\mathrm{e}}$ & $1,2(1)$ & 1 & 1 & 1 & $1-3(1)$ & - & - & - \\
\hline 12 & $1-4(2)$ & - & - & - & - & - & - & - & - & - & - \\
\hline 13 & - & $0,1^{\mathrm{c}}$ & - & - & - & - & - & - & - & - & - \\
\hline 14 & - & - & - & 1 & 1 & 1 & 1 & 1 & $1,2(1)$ & - & - \\
\hline
\end{tabular}

${ }^{\mathrm{a}}$ Main branches; ${ }^{\mathrm{b}}$ seta 10 of Reinert (1973); ${ }^{\mathrm{c}}$ usually represented by an empty alveolus; ${ }^{\mathrm{d}}$ normally absent; ${ }^{\mathrm{e}}$ often absent or represented by an empty alveolus. 

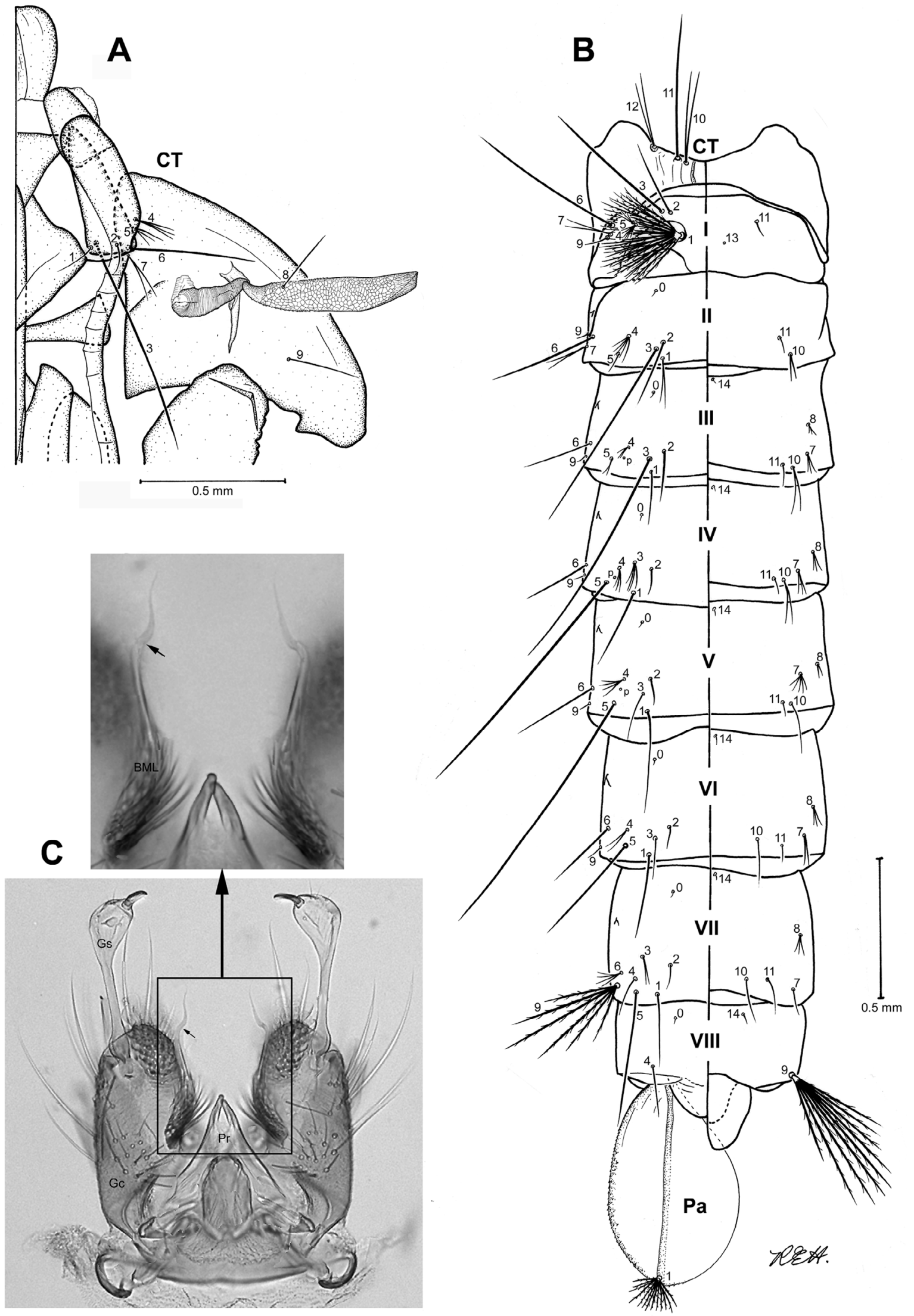

FIGURE 1. Pupa and male genitalia of Bothaella manhi: A, pupa, left side of cephalothorax, dorsal to right; B, pupa, dorsal (left) and ventral (right) aspects of metathorax and abdomen; C, male genitalia, dorsal aspect (the sigmoidal seta, arrow, is diagnostic for the species). BML, basal mesal lobe; CT, cephalothorax; Gc, gonocoxite; Gs, gonostylus; Pa, paddle; Pr, proctiger; I-VIII, abdominal segments I-VIII; 1-14, setal numbers for specified areas, e.g. seta 3-I. 
Larva, fourth-instar. Character and positions of setae as illustrated in Fig. 2; numbers of branches in Table 2. Head: Round in dorsal view, length about $0.70 \mathrm{~mm}$, width about $0.75 \mathrm{~mm}$; moderately tanned, collar and dorsomentum more darkly tanned; median labral plate very narrow, indistinct; hypostomal suture complete to posterior tentorial pit, with short extension posteriorly to collar; dorsomentum with 10 or 11 teeth on either side of median tooth. Seta 4-C relatively long, with 4-6(4) simple branches (distinction from $B o$. alongi); seta 9-C inserted posterior to level of 8-C; seta 13-C inserted much closer to 12-C than to 11-C; seta 14-C strongly developed, longer than labiogula, with 4 aciculate branches; seta 6-Mx stellate, with 4 aciculate branches. Antenna: Cylindrical, moderately tanned, surface smooth; length $0.26 \mathrm{~mm}$. Seta 1-A double, simple, length about twice diameter of antenna, inserted beyond mid-length of antenna; setae 2,3-A inserted subapically. Thorax: Integument hyaline, smooth. Setae 0,1,3,8,13,14-P, 13-M and 5,8-T somewhat stellate (not obvious in Fig. 2), minutely spiculate; setae 14-M and 13-T distinctly stellate, minutely spiculate; setae 1-3-P not borne on a setal support plate; seta 2-P single, longer than 1,3,4-P; seta 13-P more strongly developed than 14-P, similar to 8-P; 5-T also relatively strongly developed, similar to 8-T. Abdomen: Setae 1,5-I-VIII, 2,13-I-VII, 9-II-VII, 4,11-I and 1,5-VIII well developed, distinctly stellate, minutely aciculate; seta 1-V-VII with one branch distinctly longer than the others; seta 2-I-VII inserted directly anterior to slightly anterolateral to seta 1 ; seta 3-I-VI single, moderately long, 3-VII short, double or triple, similar to 4II-V; seta 4-VI-VIII single, similar to 3-I-VI; seta 6-I-VI long, branched, aciculate; seta 7-I,II similar to 6I,II but with fewer branches, 7-III-VI much shorter than 7-I,II, 7-III-V usually with 4 or 5 branches (range 36), 7-VI single; 12-I absent; seta 9-I dissimilar to 9-II-VII, double; seta 8-II-V single, 8-VI double or triple. Segment VIII: Comb comprised of 16-24 evenly fringed scales in 2 irregular rows. Seta 3-VIII noticeably much longer than 1,5-VIII, reaching to or beyond most distal pecten spine. Siphon: Tapered and slightly bent anteriorly beyond seta 1-S; lightly tanned basal to seta 1-S, progressively darker from seta 1-S to apex; length about $1.10 \mathrm{~mm}$, width at midlength about $0.17 \mathrm{~mm}$, index about 6.5 ; acus present, small, attached to base of siphon; pecten comprised of 12-18 close-set spines with 3-5 ventral denticles on proximal half, most distal denticle largest, borne at midlength. Seta 1-S inserted distal to pecten, length about twice diameter of siphon, with 3 aciculate branches. Segment X: Saddle incomplete, moderately pigmented, with cluster of 6 or 7 relatively large spines on dorsoposterior margin; dorsal length about $0.25 \mathrm{~mm}$; siphon/saddle index about 4.4; acus absent. Seta 1-X well developed, double, inserted on and much longer than saddle; ventral brush (seta 4$\mathrm{X}$ ) with 4 pairs of setae on grid (with transverse and lateral bars), anterior setae single, posterior setae double. Dorsal and ventral anal papillae equally long, longer than saddle, length about $0.5 \mathrm{~mm}$, relatively thick proximally and tapered distally.

Molecular characterization. The dataset for the region coding for part of the COI gene comprised 658 bp from 61 individuals, and for the COII region the dataset comprised 702 bp from 59 of the same individuals (Table 3). These correspond to positions 1464-2121 and 3027-3728 of Anopheles quadrimaculatus Say (GenBank accession number NC_000875), respectively. No insertions or deletions were identified and sequence alignment was unambiguous since there was no length variation in the amplified gene regions.

Among Culicidae collected in Thailand and Vietnam, the two gene regions showed similar levels of interspecific variation in uncorrected p-distance, with maximum interspecific sequence divergence of $20 \%$ between Culex fuscocephala Theobald and Toxorhynchites splendens (Wiedemann) for COI and $21 \%$ between Mansonia bonneae Edwards and Uranotaenia lateralis Ludlow for COII. The AT richness of the COI and COII fragments was 68.3 and $75.8 \%$, respectively, in agreement with previous work and reflecting the uniquely high AT content of mitochondrial genomes of higher Diptera (Jermiin \& Crozier, 1994). Intraspecific uncorrected pairwise distances were less than $2 \%$ in the majority of cases, including variation within each Bothaella species, and there was on average $9 \%$ interspecific divergence between sequences obtained from individuals representing Bo. helenae and Bo. manhi, supporting the formal naming and description of the latter species. 


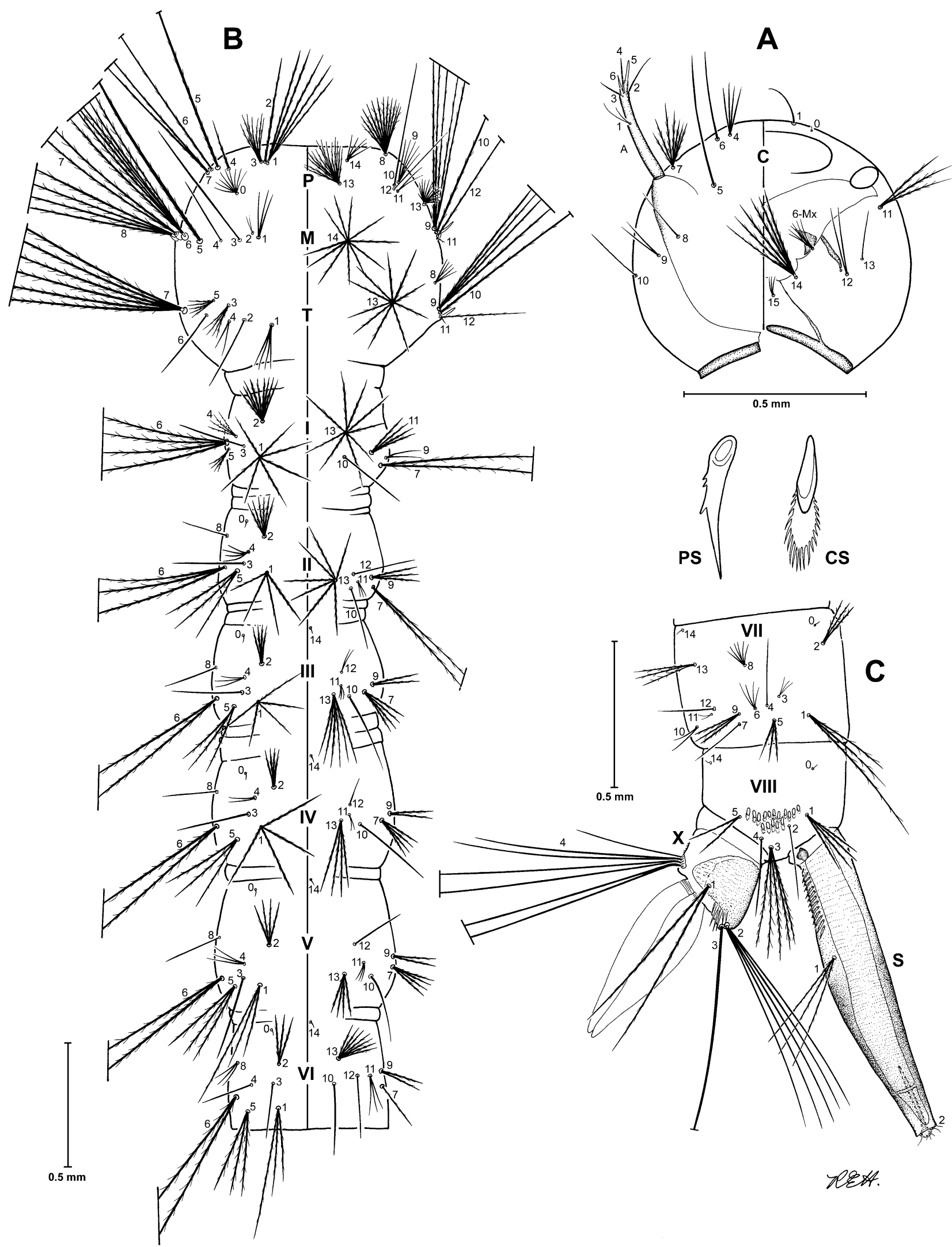

FIGURE 2. Fourth-instar larva of Bothaella manhi: A, head, dorsal (left) and ventral (right) aspects of left side; B, thorax and abdominal segments I-VI, dorsal (left) and ventral (right) aspects of left side; C, abdominal segments VII-X, left side. A, antenna; C, cranium; CS, comb scale; P, prothorax; PS, pecten spine; M, mesothorax; S, siphon; T, metathorax; I-VIII and X, abdominal segments I-VIII and X; 1-15, setal numbers for specified areas, e.g. seta 5-C. 


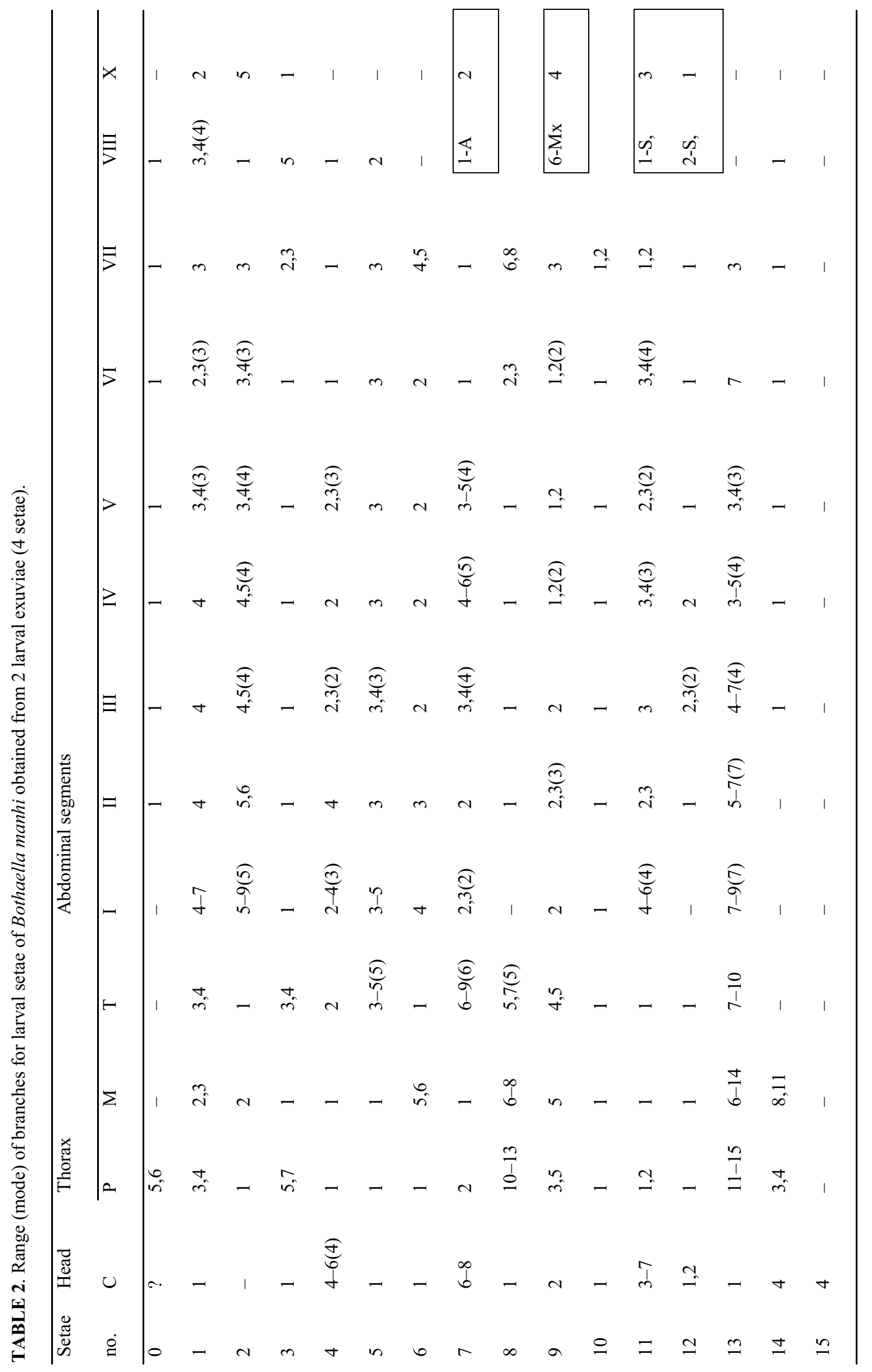

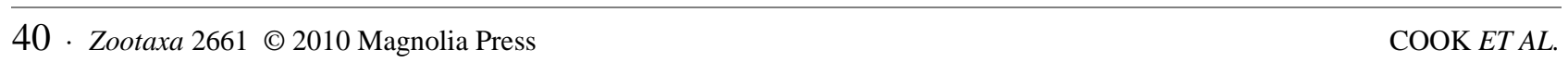


Table 3: GenBank accession numbers and collection data for specimens included in the phylogenetic analyses.

\begin{tabular}{|c|c|c|c|c|c|c|c|c|c|c|}
\hline Genus & Species & $\begin{array}{l}\text { Specimen } \\
\text { number }\end{array}$ & $\begin{array}{l}\text { COI } \\
\text { accession } \\
\text { number }\end{array}$ & $\begin{array}{l}\text { COII } \\
\text { accession } \\
\text { number }\end{array}$ & Date collected & Latitude & Longitude & $\begin{array}{l}\text { Collecting } \\
\text { method }\end{array}$ & Province & Locality* \\
\hline Bothaella & helenae & VN20-4 & HQ398919 & HQ398985 & $05 / 07 / 2000$ & - & - & D-IR & $\mathrm{NB}$ & Cuc Phuong NP \\
\hline Bothaella & helenae & VN20-22 & HQ398918 & HQ398983 & $05 / 07 / 2000$ & - & - & D-IR & NB & Cuc Phuong NP \\
\hline Bothaella & helenae & VN21-14 & HQ398920 & HQ398986 & $05 / 07 / 2000$ & - & - & D-IR & NB & Cuc Phuong NP \\
\hline Bothaella & helenae & VN21-16 & HQ398921 & HQ398987 & $05 / 07 / 2000$ & - & - & D-IR & NB & Cuc Phuong NP \\
\hline Bothaella & helenae & VN21-55 & HQ398914 & HQ398993 & $05 / 07 / 2000$ & - & - & D-IR & NB & Cuc Phuong NP \\
\hline Bothaella & helenae & VN21-76 & HQ398923 & HQ398996 & $05 / 07 / 2000$ & - & - & D-IR & NB & Cuc Phuong NP \\
\hline Bothaella & helenae & VN21-53 & HQ398922 & HQ398991 & $05 / 07 / 2000$ & - & - & D-IR & NB & Cuc Phuong NP \\
\hline Bothaella & helenae & VN104-7 & HQ398915 & HQ398975 & $19 / 08 / 2008$ & N 20,14.801 & E $105,42.900$ & BPA & NB & Cuc Phuong NP \\
\hline Bothaella & helenae & VN105-2 & HQ398916 & HQ398976 & $19 / 08 / 2008$ & N 20,19.242 & E $105,37.684$ & $\mathrm{BPA}$ & NB & Cuc Phuong NP \\
\hline Bothaella & helenae & VN113-17 & HQ398917 & HQ398978 & $20 / 08 / 2008$ & N $20,14.753$ & E $105,42.934$ & $\mathrm{BPA}$ & NB & Cuc Phuong NP \\
\hline Bothaella & kleini & VN104-8 & HQ398913 & $\mathrm{NA}$ & $19 / 08 / 2008$ & N $20,14.801$ & E $105,42.900$ & $\mathrm{BPA}$ & NB & Cuc Phuong NP \\
\hline Bothaella & kleini & VN105-1 & HQ398911 & NA & $19 / 08 / 2008$ & N 20,19.242 & E $105,37.684$ & $\mathrm{BPA}$ & NB & Cuc Phuong NP \\
\hline Bothaella & kleini & VN122-1 & HQ398912 & HQ398979 & $20 / 08 / 2008$ & N $20,15.767$ & E $105,42.964$ & BPA & NB & Cuc Phuong NP \\
\hline Bothaella & manhi & VN20-1 & HQ398924 & HQ398981 & $05 / 07 / 2000$ & - & - & D-IR & NB & Cuc Phuong NP \\
\hline Bothaella & manhi & VN20-18 & HQ398933 & HQ398980 & $05 / 07 / 2000$ & - & _- & D-IR & NB & Cuc Phuong NP \\
\hline Bothaella & manhi & VN20-21 & HQ398927 & HQ398982 & $05 / 07 / 2000$ & - & - & D-IR & NB & Cuc Phuong NP \\
\hline Bothaella & manhi & VN20-23 & HQ398928 & HQ398984 & $05 / 07 / 2000$ & - & - & D-IR & NB & Cuc Phuong NP \\
\hline Bothaella & manhi & VN21-48 & HQ398929 & HQ398988 & $05 / 07 / 2000$ & - & - & D-IR & NB & Cuc Phuong NP \\
\hline Bothaella & manhi & VN21-50 & HQ398934 & HQ398989 & $05 / 07 / 2000$ & - & - & D-IR & NB & Cuc Phuong NP \\
\hline Bothaella & manhi & VN21-52 & HQ398930 & HQ398990 & $05 / 07 / 2000$ & - & - & D-IR & NB & Cuc Phuong NP \\
\hline Bothaella & manhi & VN21-54 & HQ398931 & HQ398992 & $05 / 07 / 2000$ & - & - & D-IR & NB & Cuc Phuong NP \\
\hline Bothaella & manhi & VN21-59 & HQ398932 & HQ398994 & $05 / 07 / 2000$ & - & - & D-IR & NB & Cuc Phuong NP \\
\hline Bothaella & manhi & VN21-67 & HQ398925 & HQ398995 & $05 / 07 / 2000$ & - & - & D-IR & NB & Cuc Phuong NP \\
\hline Bothaella & manhi & VN113-16 & HQ398926 & HQ398977 & $20 / 08 / 2008$ & N 20,14.753 & E $105,42.934$ & $\mathrm{BPA}$ & NB & Cuc Phuong NP \\
\hline Anopheles & minimus & VN101-4 & HQ398936 & HQ398965 & $18-19 / 08 / 2008$ & N 20,15.547 & E $105,42.389$ & $\mathrm{LT}+$ dry ice & NB & Cuc Phuong NP \\
\hline Anopheles & minimus & VN101-5 & HQ398935 & HQ398966 & $18-19 / 08 / 2008$ & N 20,15.547 & E $105,42.389$ & $\mathrm{LT}+$ dry ice & NB & Cuc Phuong NP \\
\hline Anopheles & minimus & VN101-6 & HQ398937 & HQ398967 & $18-19 / 08 / 2008$ & N 20,15.547 & E $105,42.389$ & $\mathrm{LT}+$ dry ice & NB & Cuc Phuong NP \\
\hline Armigeres & subalbatus & VN103-2 & HQ398904 & HQ398969 & $19 / 08 / 2008$ & N $20,14.753$ & E $105,42.935$ & $\mathrm{BPA}$ & NB & Cuc Phuong NP \\
\hline Armigeres & subalbatus & VN103-3 & HQ398905 & HQ398970 & $19 / 08 / 2008$ & N $20,14.753$ & E $105,42.935$ & $\mathrm{BPA}$ & NB & Cuc Phuong NP \\
\hline Armigeres & subalbatus & VN103-4 & HQ398906 & HQ398971 & $19 / 08 / 2008$ & N $20,14.753$ & E $105,42.935$ & $\mathrm{BPA}$ & NB & Cuc Phuong NP \\
\hline Armigeres & subalbatus & VN103-5 & HQ398907 & HQ398972 & $19 / 08 / 2008$ & N $20,14.753$ & E $105,42.935$ & BPA & NB & Cuc Phuong NP \\
\hline Armigeres & subalbatus & TH148-26 & HQ398908 & HQ398939 & $13 / 08 / 2008$ & N $18,45.058$ & E $098,56.377$ & BPA & $\mathrm{CM}$ & Mae Hae \\
\hline Armigeres & subalbatus & TH148-27 & HQ398903 & HQ398940 & $13 / 08 / 2008$ & N $18,45.058$ & E $098,56.377$ & $\mathrm{BPA}$ & $\mathrm{CM}$ & Mae Hae \\
\hline Culex & bitaeniorhynchus & VN100-1 & HQ398899 & HQ398964 & $18-19 / 08 / 2008$ & N $20,15.385$ & E $105,42.539$ & $\mathrm{LT}+$ dry ice & NB & Cuc Phuong NP \\
\hline Culex & bitaeniorhynchus & TH158-4 & HQ398899 & HQ398964 & $14 / 08 / 2008$ & N $18,49.979$ & E $099,01.795$ & $\mathrm{LT}+$ dry ice & $\mathrm{CM}$ & Amphoe San Sai \\
\hline Culex & fuscocephala & TH149-4 & HQ398889 & HQ398951 & $13 / 08 / 2008$ & N $18,45.058$ & E $098,56.377$ & $\mathrm{BPA}$ & $\mathrm{CM}$ & Mae Hae \\
\hline Culex & fuscocephala & TH149-5 & HQ398891 & HQ398952 & $13 / 08 / 2008$ & N $18,45.058$ & E $098,56.377$ & BPA & $\mathrm{CM}$ & Mae Hae \\
\hline Culex & fuscocephala & TH149-8 & HQ398890 & HQ398953 & $13 / 08 / 2008$ & N $18,45.058$ & E $098,56.377$ & BPA & $\mathrm{CM}$ & Mae Hae \\
\hline Culex & fuscocephala & TH149-9 & HQ398954 & $13 / 08 / 08$ & $13 / 08 / 2008$ & N $18,45.058$ & E $098,56.377$ & $\mathrm{BPA}$ & $\mathrm{CM}$ & Mae Hae \\
\hline Culex & fuscocephala & TH149-10 & HQ398887 & HQ398946 & $13 / 08 / 2008$ & N $18,45.058$ & E $098,56.377$ & $\mathrm{BPA}$ & $\mathrm{CM}$ & Mae Hae \\
\hline Culex & fuscocephala & TH149-18 & HQ398888 & HQ398949 & $13 / 08 / 2008$ & N $18,45.058$ & E $098,56.377$ & $\mathrm{BPA}$ & $\mathrm{CM}$ & Mae Hae \\
\hline Culex & gelidus & TH148-2 & HQ398894 & HQ398941 & $13 / 08 / 2008$ & N $18,45.058$ & E $098,56.377$ & $\mathrm{BPA}$ & $\mathrm{CM}$ & Mae Hae \\
\hline Culex & gelidus & TH148-3 & HQ398895 & HQ398942 & $13 / 08 / 2008$ & N $18,45.058$ & E $098,56.377$ & BPA & $\mathrm{CM}$ & Mae Hae \\
\hline Culex & gelidus & TH148-4 & HQ398892 & HQ398943 & $13 / 08 / 2008$ & N $18,45.058$ & E $098,56.377$ & BPA & $\mathrm{CM}$ & Mae Hae \\
\hline Culex & gelidus & TH148-6 & HQ398893 & HQ398944 & $13 / 08 / 2008$ & N $18,45.058$ & E $098,56.377$ & $\mathrm{BPA}$ & $\mathrm{CM}$ & Mae Hae \\
\hline Culex & nigropunctatus & TH149-19 & HQ398882 & HQ398950 & $13 / 08 / 2008$ & N $18,45.058$ & E $098,56.377$ & $\mathrm{BPA}$ & $\mathrm{CM}$ & Mae Hae \\
\hline Culex & quinquefasciatus & TH148-8 & HQ398883 & HQ398945 & $13 / 08 / 2008$ & N $18,45.058$ & Е $098,56.377$ & $\mathrm{BPA}$ & $\mathrm{CM}$ & Mae Hae \\
\hline Culex & rubithoracis & TH151-1 & HQ398884 & HQ398959 & $14 / 08 / 2008$ & N $18,41.963$ & E $099,08.724$ & $\mathrm{BPA}$ & $\mathrm{L}$ & Amphoe Ban Thi \\
\hline Culex & tritaeniorhynchus & TH148-19 & HQ398885 & HQ398938 & $13 / 08 / 2008$ & N $18,45.058$ & E $098,56.377$ & $\mathrm{BPA}$ & $\mathrm{CM}$ & Mae Hae \\
\hline Lutzia & fuscana & TH150-7 & HQ398896 & HQ398957 & $13 / 08 / 2008$ & N $18,44.808$ & E $098,57.003$ & BPA & $\mathrm{CM}$ & Mae Hae \\
\hline Lutzia & fuscana & TH150-8 & HQ398897 & HQ398958 & $13 / 08 / 2008$ & N $18,44.808$ & E $098,57.003$ & $\mathrm{BPA}$ & $\mathrm{CM}$ & Mae Hae \\
\hline Mansonia & bonneae & TH149-14 & HQ398878 & HQ398947 & $13 / 08 / 2008$ & N $18,45.058$ & E $098,56.377$ & $\mathrm{BPA}$ & $\mathrm{CM}$ & Mae Hae \\
\hline Mansonia & bonneae & TH149-15 & HQ398879 & HQ398948 & $13 / 08 / 2008$ & N $18,45.058$ & E $098,56.377$ & $\mathrm{BPA}$ & $\mathrm{CM}$ & Mae Hae \\
\hline Mansonia & uniformis & TH158-1 & HQ398880 & HQ398960 & $14 / 08 / 2008$ & N $18,49.979$ & E $099,01.795$ & $\mathrm{LT}+$ dry ice & $\mathrm{CM}$ & Amphoe San Sai \\
\hline Neomelaniconion & lineatopenne & TH150-1 & HQ398909 & HQ398955 & $13 / 08 / 2008$ & N $18,44.808$ & Е $098,57.003$ & BPA & $\mathrm{CM}$ & Mae Hae \\
\hline Neomelaniconion & lineatopenne & TH150-2 & HQ398910 & HQ398956 & $13 / 08 / 2008$ & N $18,44.808$ & E $098,57.003$ & BPA & $\mathrm{CM}$ & Mae Hae \\
\hline Stegomyia & albopicta & VN103-9 & HQ398902 & HQ398973 & $19 / 08 / 2008$ & N 20,14.753 & E $105,42.935$ & $\mathrm{BPA}$ & NB & Cuc Phuong NP \\
\hline Stegomyia & albopicta & VN103-10 & HQ398900 & HQ398968 & $19 / 08 / 2008$ & N $20,14.753$ & E $105,42.935$ & BPA & NB & Cuc Phuong NP \\
\hline Stegomyia & albopicta & VN104-2 & HQ398901 & HQ398974 & $19 / 08 / 2008$ & N $20,14.801$ & E $105,42.900$ & BPA & NB & Cuc Phuong NP \\
\hline Toxorhynchites & splendens & TH162-3 & HQ398877 & HQ398962 & $16 / 08 / 2008$ & N $18,54.087$ & E $098,49.821$ & BPA & $\mathrm{CM}$ & Amphoe San Sai \\
\hline Uranotaenia & lateralis & TH162-4 & HQ398881 & HQ398963 & $16 / 08 / 2008$ & N $18,54.087$ & E $098,49.821$ & BPA & $\mathrm{CM}$ & Amphoe San Sai \\
\hline
\end{tabular}

$\mathrm{BPA}=$ Backpack aspirator; $\mathrm{CM}=$ Chiang Mai; D-IR $=$ Dropper - Individual rearing of larva or pupa; $\mathrm{L}=$ Lamphun $\mathrm{LT}=$ Light trap; $\mathrm{NB}=\mathrm{Ninh} \mathrm{Binh} ; \mathrm{NP}=\mathrm{National}$ Park; $\mathrm{TH}=\mathrm{Thailand} ; \mathrm{VN}=$ Vietnam.

The maximum likelihood trees for COI and COII are shown in Figs 3 and 4, respectively. In the case of both trees, all species-level clades were supported by high (100\%) posterior probabilities. The only exception comprised two specimens representing Ma. bonneae with posterior probabilities of $75 \%$ in the phylogeny based on the COI region but $100 \%$ in the phylogeny based on the COII region. Importantly, in some cases, sequences for a given species originate from wild mosquitoes from widely dispersed geographic locations, namely the Armigeres subalbatus (Coquillett) and Culex bitaeniorhynchus Giles lineages, or from different sampling years, for example 2000 and 2008 within both Bo. manhi and Bo. helenae, but despite this, sequences still group strongly according to species. In addition, currently accepted species groupings are well resolved in ML phylogenies of both the COI and COII regions. Taken together, the results strongly support (1) the utility of mtDNA for species-level identification of Culicidae and (2) the formal naming and description of Bo. manhi and its inferred sister relationship to Bo. helenae, with Bo. kleini forming their near relative. Interestingly, in contrast, phylogenetic structure at the subfamily level for both genes was either absent or of limited support, reflected by posterior probabilities of less than $50 \%$ in general and in agreement with previous studies (Cook et al., 2005). 


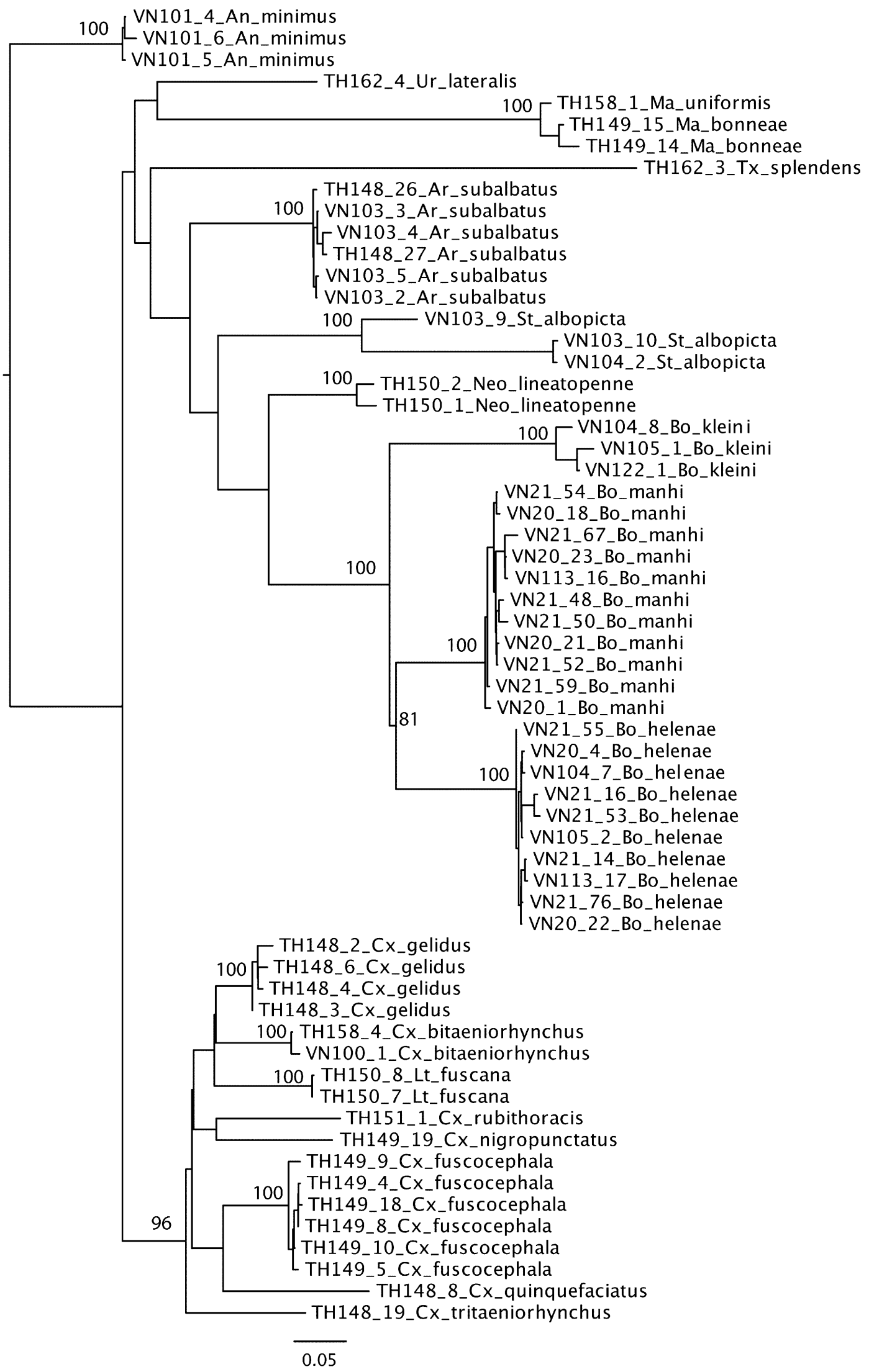

FIGURE 3. Bayesian phylogeny of the "barcode" region of the COI nucleotide dataset of the Culicidae collected in Vietnam and Thailand. Posterior probabilities (percent) of 80 or higher are shown for main clades only. See Table 3 for specimen collection data and accession numbers. All horizontal branch lengths are drawn to a scale of substitutions per site. The tree is rooted on Anopheles minimus Theobald. 


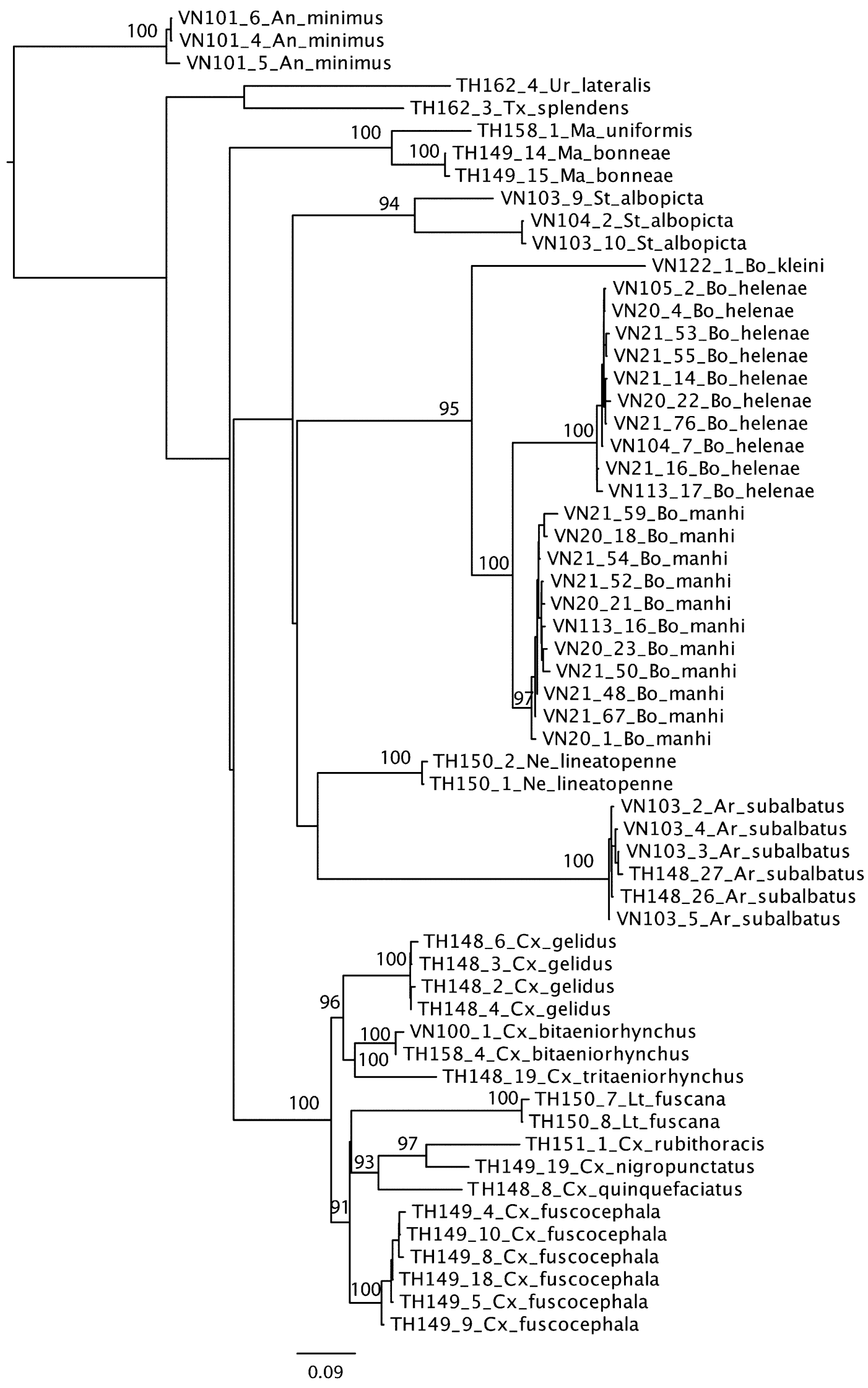

FIGURE 4. Bayesian phylogeny of the COII nucleotide dataset of the Culicidae collected in Vietnam and Thailand. Posterior probabilities (percent) of 80 or higher are shown for main clades only. See Table 3 for specimen collection data and accession numbers. All horizontal branch lengths are drawn to a scale of substitutions per site. The tree is rooted on An. minimus. 
Systematics. Diagnostic and differential features that distinguish the three species of Bothaella with banded hindtarsi that are known to occur in Vietnam are listed in Table 4. In view of these differences, and characters that distinguish the five species of Bothaella, Bo. manhi is most similar to Bo. helenae, the adult females and pupae of which are inseparable. This is also supported by ML phylogenetic analyses of DNA sequences, which suggests that Bo. manhi + Bo. helenae forms a sister group to Bo. kleini (Figs 3 and 4).

Bionomics. The immature stages of Bo. manhi were collected from very small holes in limestone outcrops in rainforest. The holes contained clear fresh water and were heavily shaded. Adult females were collected resting on vegetation in forest and a primate sanctuary. Indirect evidence suggests that females may feed on primates and other mammals. Nothing else is known about the bionomics of the species.

TABLE 4. Comparison of diagnostic and differential characters observed in three species of Bothaella with banded hindtarsi that occur in northern Vietnam. Bothaella kleini also occurs in northern Vietnam but its hindtarsi are entirely dark-scaled.

\begin{tabular}{|c|c|c|c|c|}
\hline & Character & Bo. alongi ${ }^{1}$ & Bo. helenae ${ }^{2}$ & Bo. manhi \\
\hline \multirow[t]{7}{*}{ Adults } & Postgena & Dark-scaled & With silvery scale-patch & With silvery scale-patch \\
\hline & Postpronotal setae & 3 & 3 or 4 & 2 or 3 \\
\hline & $\begin{array}{l}\text { Upper mesokatepisternal } \\
\text { setae }\end{array}$ & 2 or 3 & 3 & 2 or 3 \\
\hline & Forecoxa & $\begin{array}{l}\text { Dark-scaled anteriorly } \\
\text { at apex }\end{array}$ & $\begin{array}{l}\text { Patch of silvery scales } \\
\text { anteriorly at apex }\end{array}$ & $\begin{array}{l}\text { Patch of silvery scales } \\
\text { anteriorly at apex }\end{array}$ \\
\hline & Hindfemur & $\begin{array}{l}\text { Proximal } 0.70 \text { pale- } \\
\text { scaled with dorsal line } \\
\text { of dark scales }\end{array}$ & $\begin{array}{l}\text { Proximal } 0.75-0.80 \text { pale- } \\
\text { scaled with dorsal line of } \\
\text { dark scales }\end{array}$ & $\begin{array}{l}\text { Proximal } 0.50-0.60 \text { pale- } \\
\text { scaled, usually without } \\
\text { trace of dorsal dark scales }\end{array}$ \\
\hline & Wing vein $R 2+3$ & Longer than vein $\mathrm{R} 2$ & Shorter than vein $\mathrm{R} 2$ & Shorter than vein R2 \\
\hline & $\begin{array}{l}\text { Basal mesal lobe }(\hat{\sigma} \\
\text { genitalia) }\end{array}$ & Unknown & Without long sigmoid seta & With long sigmoid seta \\
\hline \multirow[t]{19}{*}{ Larvae } & Seta 4-C & 10-14 branches & 4-8(4) branches & 4-6(4) branches \\
\hline & Seta $14-C$ & 5-9 branches & 5-8(6) branches & 4 branches \\
\hline & Seta 6-Mx & 6-11 branches & 9-15 branches & 4 branches \\
\hline & Seta 0-P & 15,16 branches & $8-14(9)$ & 5,6 branches \\
\hline & Seta 3-P & 15-17 branches & 11-15(12) branches & 5,7 branches \\
\hline & Seta $1-M$ & Multi-branched & 5-7(6) branches & 2,3 branches \\
\hline & Seta $1-\mathrm{T}$ & 22-24 branches & $5-12(6)$ & 3,4 branches \\
\hline & Seta 8-T & 11 branches & 10-16(11) branches & 4-6(4) branches \\
\hline & Seta 1-II-IV & Multi-branched & $6-10$ branches & 4 branches \\
\hline & Seta 6-I & 7-9 branches & $3,4(3)$ branches & 4 branches \\
\hline & Seta 6-II & 7,8 branches & 3 branches & 3 branches \\
\hline & Seta 4-III & Stellate & 5-8(5) branches & $2,3(3)$ branches \\
\hline & Seta 4-IV & Stellate & 4-9(6) branches & 2 branches \\
\hline & Seta 9-III-VI & $?$ & 3-6(3,4) branches & $1,2(2)$ branches \\
\hline & Seta 5-IV-VII & Stellate & 4,5(4) branches & 3 branches \\
\hline & Seta 1-VIII & 14-17 branches & 8-11(8) branches & $3,4(4)$ branches \\
\hline & Seta 5-VIII & 6 branches & 3-7(5) branches & 2 branches \\
\hline & Pecten spines & $9-11$ & $13-16$ & $12-18$ \\
\hline & Siphon (index) & Short (2.2) & Long (ca. 6.5) & Long (4.1-4.9) \\
\hline
\end{tabular}

${ }^{1}$ Data from Reinert \& Harbach (2006); ${ }^{2}$ data from Reinert (1973). 
Distribution. Bothaella manhi is only known from the Cuc Phuong National Park of Ninh Binh Province in northern Vietnam.

Etymology. Bothaella manhi is dedicated to the memory of Dr Nguyen Duc Manh, our respected colleague, co-researcher and friend who died unexpectedly while this manuscript was in preparation. Dr Manh was Head of the Department of Molecular Biology, National Institute of Malariology, Parasitology and Entomology (NIMPE) in Hanoi prior to his death on 7 July 2010. He will be remembered for his exuberant interest and participation in entomological field studies and his contributions to mosquito taxonomy. Dr Manh (Fig. 5) arranged and participated in the visits to the Cuc Phuong National Park that resulted in the discovery of the new species named in his honour here. (NB: In Vietnam, as in many Asian countries, the family name is placed before the individual's given name; hence, individuals are addressed by their given name.)

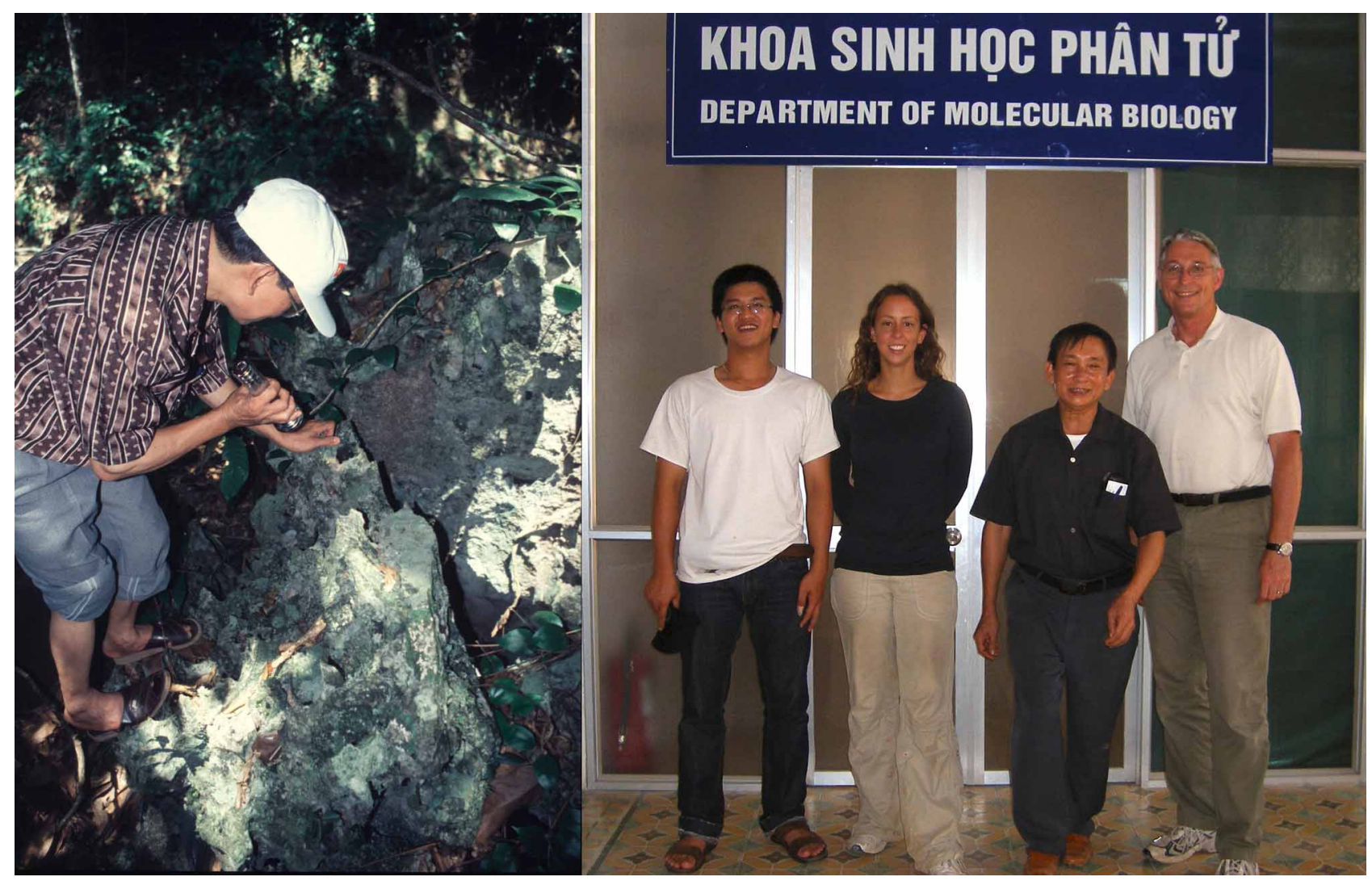

FIGURE 5. Left: Dr Nguyen Duc Manh examining a rock-hole habitat of Bothaella larvae during mosquito surveys conducted in the Cuc Phuong National Park in July 2000. Right: Dr Manh (second from right) with field assistant Nguyen Hong Hanh (far left), Shelley Cook and Ralph Harbach outside the Molecular Biology Laboratory of the National Institute of Malariology, Parasitology and Entomology prior to field studies conducted in the same park in August 2008.

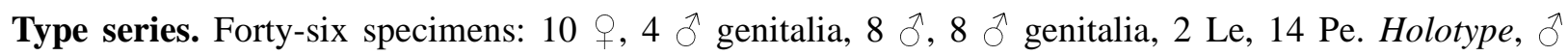
(VN21-49), with Pe and dissected genitalia on microscope slides, VIETNAM: Ninh Binh Province, Cuc Phuong National Park, rock hole, 5 July 2000 (Harbach et al.). Paratypes (same locality and collectors as holotype), 2 \&ePe (VN20-23, VN21-51); 8 Pe (VN20-1, -2 with dissected genitalia on microscope slides; VN21-48, -50, -67; VN21-59 with dissected genitalia on microscope slide; VN47-2); 1 \& (VN47-2); 7 Pe (VN20-16, -18, -19, -21 and VN21-49, -52, -54 with dissected genitalia on microscope slides); 1 (VN1152), same locality, forest edge near stream, and $1 \hat{O}$ (VN113-16), Endangered Primate Sanctuary, both with dissected genitalia on microscope slides, 20 August 2008 (Cook et al.). Specimens for which DNA sequence are available are indicated in Table 3. The type series is deposited in the Natural History Museum (BMNH), London. 


\section{Acknowledgements}

We are grateful to field workers from the National Institute of Malariology, Parasitology and Entomology for their participation in the field work; to Luis Hernandez, Department of Entomology, Natural History Museum (NHM), London, for taking the photomicrographs used to prepare Fig. 1C. The work in Vietnam and Thailand was conducted as part of a Sir Henry Wellcome Trust Fellowship (Grant number 082744) awarded to Shelley Cook for her study entitled "Emerging arboviruses: the effect of vector and host biology and genetic diversity on the emergence and maintenance of the flaviviruses". The field work was also supported by funds provided from the Department of Entomology Investment Fund (NHM, London).

\section{References}

Cook, S., Bennett, S.N., Holmes, E.C., De Chesse, R., Moureau, G. \& de Lamballerie, X. (2006) Isolation of a new strain of the flavivirus cell fusing agent virus in a natural mosquito population from Puerto Rico. Journal of General Virology, 87, 735-748.

Cook, S., Diallo, M., Sall, A.A., Cooper, A. \& Holmes, E.C. (2005) Mitochondrial markers for molecular identification of Aedes mosquitoes (Diptera: Culicidae) involved in transmission of arboviral disease in West Africa. Journal of Medical Entomology, 42, 19-28.

Dong, X., Zhou, H. \& Dong, L. (1999) A new species and a new Chinese record of the subgenus Bothaella of genus Aedes (Diptera: Culicidae) [in Chinese]. Entomotaxonomia, 21, 133-137.

Drummond, A.J. \& Rambaut, A. (2007) BEAST: Bayesian evolutionary analysis by sampling trees. BMC Evolutionary Biology, 7, 214.

Edgar, R.C. (2004) MUSCLE: multiple sequence alignment with high accuracy and high throughput. Nucleic Acids Research, 32, 1792-1797.

Folmer, O., Black, M., Hoeh, W., Lutz, R. \& Vrijenhoek, R. (1994) DNA primers for amplification of mitochondrial cytochrome c oxidase subunit I from diverse metazoan invertebrates. Molecular Marine Biology and Biotechnology, 3, 294-297.

Galliard, H. \& Ngu, D.-V. (1947) Culicinés du Tonkin I. Liste des espèces et leur répartition. Annales de Parasitologie Humaine et Comparée, 22, 75-80.

Huelsenbeck, J.P. \& Ronquist, F. (2001) MRBAYES: Bayesian inference of phylogeny. Bioinformatics, 17, 754-755.

Jermiin, L.S. \& Crozier, R.H. (1994) The cytochrome b region in the mitochondrial DNA of the ant Tetraponera rufoniger: sequence divergence in Hymenoptera may be associated with nucleotide content. Journal of Molecular Evolution, 38, 282-294.

Liu, H. \& Bechenback, A.T. (1992) Evolution of the mitochondrial cytochrome oxidase II gene among ten orders of insects. Molecular Phylogenetics and Evolution, 1, 41-52.

Reinert, J.F. (1973) Contributions to the mosquito fauna of Southeast Asia. -XIX. Bothaella, a new subgenus of Aedes Meigen. Contributions of the American Entomological Institute, 10(3), 1-51.

Reinert, J.F., Harbach, R.E. \& Huong, V.D. (2006) Aedes (Finlaya) alongi Galliard and Ngu (Diptera: Culicidae: Aedini): description of the female, redescription of the fourth-instar larva, and reassignment to genus Bothaella Reinert. Zootaxa, 1135, 49-55. 